\title{
Controlling NMDA Receptor Subunit Composition Using Ectopic Retention Signals
}

\author{
David Stroebel, ${ }^{1,2,3 *}$ Stéphanie Carvalho, ${ }^{1,2,3 *}$ Teddy Grand, ${ }^{1,2,3}$ Shujia Zhu, ${ }^{1,2,3}$ and Pierre Paoletti ${ }^{1,2,3}$ \\ ${ }^{1}$ Ecole Normale Supérieure, Institut de Biologie de l'École Normale Supérieure, Paris F-75005, France, ${ }^{2}$ INSERM, U1024, Paris F-75005, France, and ${ }^{3}$ Centre \\ National de la Recherche Scientifique, Unité Mixte de Recherche 8197, Paris F-75005, France
}

\begin{abstract}
Ligand-gated ion channels (LGICs) mediate fast synaptic transmission in the CNS. Typically, these membrane proteins are multimeric complexes associating several homologous subunits around a central pore. Because of the large repertoire of subunits within each family, LGICs exist in vivo as multiple subtypes that differ in subunit composition and functional properties. Establishing the specific properties of individual receptor subtypes remains a major goal in the field of neuroscience and molecular pharmacology. However, isolating specific receptor subtype in recombinant systems can be problematic because of the mixture of receptor populations. This is the case for NMDA receptors (NMDARs), a large family of tetrameric glutamate-gated ion channels that play key roles in brain physiology and pathology. A significant fraction of native NMDARs are triheteromers composed of two GluN1 subunits and two different GluN2 subunits (GluN2A-D). We developed a method based on dual retention signals adapted from G-protein-coupled GABA-B receptors allowing exclusive cell surface expression of triheteromeric rat NMDARs while coexpressed diheteromeric receptors (which contain a single type of GluN2 subunit) are retained intracellularly. Using this approach, we determined the functional properties of GluN1/GluN2A/GluN2B triheteromers, one of the most abundant NMDAR subtypes in the adult forebrain, revealing their unique gating and pharmacological attributes. We envision applicability of the retention signal approach for the study of a variety of heteromeric glutamate-gated ion channel receptors with defined subunit composition.
\end{abstract}

Key words: glutamate; ligand-gated ion channel; membrane receptor; neurotransmitter receptor; NMDA receptor; pharmacology

\section{Introduction}

Glutamate receptor ion channels (iGluRs) mediate excitatory transmission and synaptic plasticity in the brain (Traynelis et al., 2010). iGluRs exist as three main classes, AMPA, kainate, and NMDA, with receptors assembling from four homologous subunits among a large repertoire. In vivo iGluRs occur predominantly as heteromers of different subunit types and isoforms. The combinatorial association of individual subunits yields a large number of receptor subtypes, thus diversifying their functional and signaling properties. This is exemplified by NMDA receptors (NMDARs), obligate heteromers typically associating two GluN1and two GluN2 subunits. The GluN1 subunit is encoded by a single gene but occurs as eight distinct splicing isoforms (GluN1-1a/b to GluN1-4a/b). Separate genes give rise to four distinct GluN2 subunits (GluN2A-D). Although the obligate GluN1 subunit is ubiquitously expressed in the CNS

\footnotetext{
Received July 4, 2014; revised Oct. 1, 2014; accepted Oct. 6, 2014.

Author contributions: D.S., S.C., T.G., S.Z., and P.P. designed research;D.S., S.C., T.G., and S.Z. performed research; D.S., S.C., T.G., S.Z., and P.P. analyzed data; D.S. and P.P. wrote the paper.

This work was supported by the Fondation pour la Recherche Médicale (Equipe FRM Grant DEQ2000326520 to P.P.) and by the French government (Investissements d'Avenir Grants ANR-10-LABX-54 MEMO LIFE and ANR-11IDEX-0001-02 PSL to Research University). We thank Andrea Yao for critical comments on the manuscript.

The authors declare no competing financial interests.

*D.S. and S.C. contributed equally to this work.

Correspondence should be addressed to Dr. Pierre Paoletti, Institut de Biologie de l'ENS, Ecole Normale Supérieure, 46 rue d'Ulm, 75005 Paris, France. E-mail: pierre.paoletti@ens.fr.

DOI:10.1523/JNEUROSCI.2736-14.2014

Copyright $\odot 2014$ the authors $\quad 0270-6474 / 14 / 3416630-07 \$ 15.00 / 0$
}

throughout development, individual GluN2 subunits have distinct expression profiles that change strikingly during development (Sanz-Clemente et al., 2013; Paoletti et al., 2013).

Because individual neurons usually express at least two different GluN2 subunits, native NMDARs can occur as diheteromers, incorporating GluN1 and two identical copies of a given GluN2 subunit, or as triheteromers, incorporating two distinct GluN2 subunits. Compelling evidence indicates that diheteromers and triheteteromers coexist within a single cell or even at a single synapse, adding to the functional diversity of the postsynaptic response (Paoletti et al., 2013). In the adult hippocampus and cortex, GluN1/GluN2A/GluN2B receptors are particularly abundant, representing a significant fraction of the total NMDAR population (Sheng et al., 1994; Chazot and Stephenson, 1997; Luo et al., 1997; Al-Hallaq et al., 2007; Gray et al., 2011; Rauner and Köhr, 2011; Tovar et al., 2013). Despite their prevalence, the functional properties of triheteromeric NMDARs remain poorly understood because of the difficulty in isolating triheteromers in expression systems. A previous study succeeded in assessing the sensitivity of recombinant triheteromers to subunit-specific allosteric inhibitors (Hatton and Paoletti, 2005). However, the approach had obvious limitations because of introduced mutations in the pore and glutamate-binding domains. We now present a more generalizable and versatile approach to isolate and characterize heterologously expressed triheteromeric NMDARs. It exploits the trafficking control system of G-protein-coupled GABA-B receptors that associate GABA-B1 and GABA-B2 sub- 
units. In this system, GABA-B1 contains a retention signal in its cytoplasmic C-terminal domain (CTD) that traps the subunit in the endoplasmic reticulum (ER). Upon association with the GABA-B2 CTD, the GABA-B1 retention signal is masked through a coiled-coil interaction, thus allowing trafficking of the GABA-B1/GABA-B2 heterodimer to the cell surface (MargetaMitrovic et al., 2000; Calver et al., 2001; Pagano et al., 2001). Brock et al. (2007) refined the system further by adding an intracellular retention signal $(\mathrm{KKXX})$ to the GABA-B2 CTD, which permits retention of GABA-B1 and GABA-B2 in isolation but reciprocal unmasking upon coexpression. By transferring this dual retention system to GluN2 subunits, we succeeded in expressing "pure" GluN1/GluN2A/GluN2B triheteromers at the surface of Xenopus oocytes, enabling direct evaluation of their functional properties. Similarities and differences with a recent work that used a similar approach (Hansen et al., 2014) are discussed.

\section{Materials and Methods}

Molecular biology. Plasmids for rodent NMDAR subunits have been described previously (Mony et al., 2011). Plasmids encoding the rat GABA-B1a and GABA-B2 subunits were generously provided by Julie Kniazeff and Jean-Philippe Pin (Montpellier, France). The r1 (TMKTGS STNNNEEEKSRLLEKENRELEKIIAEKEERVSELRHQLQSRQQLRSRR HPPTPPDPSGGLPRGPSEPPDRLSCDGSRVHLLYK) and r2 (QFTQN QKKEDSKTSTSVTSVNQASTSRLEGLQSENHRLRMKITELDKDLEEVT MQLQDTPEKKTN) tags derive from GABA-B1 and GABA-B2 CTD, respectively. Each contains a leucine zipper coiled-coil motif (underlined) followed by an ER retention signal (bold). The GluN2Ar1, GluN2Ar2, GluN2Br1, and GluN2Br2 constructs correspond to GluN2A or GluN2B subunits in which the last 602 (starting at GluN2A-I863) or 620 (starting at GluN2B-S863) residues were replaced by the $\mathrm{r} 1$ and $\mathrm{r} 2$ tag. Several other GluN2A-tagged constructs were tested but none achieved proper retention (as assessed by current measurements). In particular, "leakage" was observed when replacing GluN2A CTD by the $\mathrm{r} 1$ tag at position G873, when a flexible GAAGG sequence was added just upstream of the $\mathrm{r} 1$ tag, or when the $\mathrm{r} 1$ or $\mathrm{r} 2$ tag was inserted between positions S862 and I863. The GluN1 $\Delta \mathrm{C}$ construct contains a stop codon at position GluN1-R839. GluN1-6A corresponds to the GluN1-1a subunit with alanine mutations at positions: $\mathrm{K}_{875} \mathrm{~K}_{876} \mathrm{~K}_{877^{-}}$ $\mathrm{R}_{893} \mathrm{R}_{894} \mathrm{R}_{895}$. Plasmids encoding GluN1-2a, GluN1-3a, and GluN1-4a were generously provided by Wei Lu.

Electrophysiology. Oocytes from female Xenopus laevis were prepared, injected, and voltage clamped as described previously (Paoletti et al., 1997). Each oocyte was coinjected with a mixture of GluN1:GluN2 cDNAs $(10-70 \mathrm{ng} / \mu \mathrm{l})$ at a ratio of $1: 1$ for diheteromers and 2:1:1 for triheteromers. Data were collected using pClamp 10 and fitted using SigmaPlot 10 or Kaleidagraph 4 . The external solution contained the following (in mM): $100 \mathrm{NaCl}, 2.5 \mathrm{KCl}, 0.3 \mathrm{BaCl}_{2}, 5$ HEPES, 0.01 DTPA, $\mathrm{pH}$ 7.3. For zinc experiments, DTPA was omitted and tricine $(10 \mathrm{~mm})$ used to buffer zinc (Paoletti et al., 1997). Proton experiments were performed as in Gielen et al. (2008) and spermine experiments $(-30 \mathrm{mV}$, pH 6.5) as in Mony et al. (2011). NMDAR-mediated currents were induced by coapplication of L-glutamate and glycine (100 $\mu \mathrm{m}$ each). Unless specified, recordings were performed at $-60 \mathrm{mV}$ and at room temperature.

Glutamate deactivation kinetics experiments were performed on outside-out patches pulled from Xenopus oocytes using similar recording conditions as in Zhu et al. (2013). Currents were sampled at $5 \mathrm{kHz}$ and low-pass filtered at $2 \mathrm{kHz}$. Glutamate ( $100 \mu \mathrm{M}, 200 \mathrm{~ms})$ was applied using a multibarrel rapid solution exchanger system (RSC 160; Bio-Logic).

Pharmacology and data analysis. Glutamate and glycine doseresponse-curve (DRC) experiments were performed in the presence of $100 \mu \mathrm{M}$ of the respective coagonist. Agonist DRCs were fitted with the following Hill equation: $I_{\text {rel }}=1 /\left(1+\left(\mathrm{EC}_{50} /[A]\right)^{\mathrm{nH}}\right)$, where $I_{\text {rel }}$ is the mean current normalized to the current obtained at $100 \mu \mathrm{M}$ agonist, $[A]$ is the agonist concentration, and $\mathrm{n}_{\mathrm{H}}$ is the Hill coefficient. $\mathrm{EC}_{50}$ and $\mathrm{n}_{\mathrm{H}}$ were set as free parameters. Determination of MK-801 inhibition time constant $\left(\tau_{\text {on }}\right)$ was performed as in Mony et al. (2011). Proton DRCs were fitted as in Gielen et al. (2008) with $\mathrm{pH}_{\mathrm{IC} 50}$ and $\mathrm{n}_{\mathrm{H}}$ set as free parameters. Zinc and ifenprodil DRCs were fitted with the following Hill equation; for diheteromers: $I_{\text {rel }}=1-a /\left(1+\left(\mathrm{IC}_{50} /[C]\right)^{\mathrm{nH}}\right)$, with $a$ the maximal inhibition (Inhib . max); for triheteromers: $I_{\text {rel }}=1-[a /(1+$ $\left.\left.\left(\mathrm{IC}_{50,1} /[\mathrm{C}]\right)^{\mathrm{nH1}}\right)+(1-a) /\left(1+\left(\mathrm{IC}_{50,2} /[C]\right)^{\mathrm{nH} 2}\right)\right] . \mathrm{IC}_{50}, \mathrm{IC}_{50,1}, \mathrm{IC}_{50,2}$, $\mathrm{n}_{\mathrm{H}}, \mathrm{n}_{\mathrm{H} 1}$, and $\mathrm{n}_{\mathrm{H} 2}$ were set as free parameters. $a$ was set as a free parameter unless stated (Table 1). Note that, for triheteromers, we assume that inhibition is total at high concentrations of zinc or ifenprodil. Spermine DRCs were fitted with the following Hill equation: $I_{\text {rel }}=1+a /(1+$ $\left.\left(\mathrm{EC}_{50} /[C]\right)^{\mathrm{nH}}\right)$, where $(a+1)$ is the maximal potentiation (Pot . max). $\mathrm{EC}_{50}, a$, and $\mathrm{n}_{\mathrm{H}}$ were set as free parameters. Glutamate deactivation currents were fitted with a mono-exponential function. Error bars represent SD.

Immunoblotting. Immunoblots were performed as described previously (Mony et al., 2011) except that electrophoresis were done in reducing conditions ( $5 \% \beta$-mercaptoethanol). The following antibodies were used: anti-GluN1 antibody (1:1000, mouse monoclonal clone 54.1; Millipore), anti-GluN2B antibody (1:500, mouse monoclonal clone N59/36 or N59/20; Neuromab), anti-GABA-B1 antibody (1:500; rabbit monoclonal antibody raised against the GABA-B1 CTD; generous gift from Julie Kniazeff, Montpellier, France), anti- $\alpha$ Tubuline (1:7000, mouse monoclonal clone DM1A; Millipore), and secondary goat peroxidaseconjugated anti-mouse antibody (1:20 000, catalog \#115-035-003; Jackson ImmunoResearch).

\section{Results}

\section{Selective expression of triheteromeric NMDARs}

To selectively express triheteromeric GluN1/GluN2A/GluN2B receptors, we constructed GluN2A and GluN2B subunits with modified CTDs containing the optimized quality control system of GABA-B receptors (Brock et al., 2007). We introduced either one of two domains, coined ret 1 and ret 2 (or $\mathrm{r} 1$ and $\mathrm{r} 2$ ). The $\mathrm{r} 1$ motif contains the majority of the GABA-B1 CTD including a leucine zipper coiled-coil motif followed by a native ER retention signal RSR. The $\mathrm{r} 2$ motif comprises a fragment the GABA-B2 CTD encompassing a leucine zipper coiled-coil motif followed by an engineered dilysine KKXX ER retention motif. We constructed several versions of modified GluN2A and GluN2B subunits with $\mathrm{r} 1$ and $\mathrm{r} 2$ motifs positioned at various locations in the GluN2 CTD. Because the GluN2 CTDs are very long and likely flexible (Ryan et al., 2008), we chose not to introduce the $r 1$ and r2 motifs at the very $\mathrm{C}$ terminus of GluN2 subunits but closer to the pore region (Fig. $1 A$ ) to avoid potential recognition of $\mathrm{r} 1$ and $\mathrm{r} 2$ motifs between, rather than within, individual receptor complexes.

We first checked for proper ER retention caused by $\mathrm{r} 1$ and $\mathrm{r} 2$ motifs by expressing wild-type (wt) GluN1-1a subunit (also named GluN1 for simplicity) and individual CTD-modified GluN2A or GluN2B subunit in Xenopus oocytes. When replacing GluN2A CTD by the $\mathrm{r} 1$ motif at position GluN2A-I863 (GluN2Ar1 subunit), no or very tiny ( $\leq 10 \mathrm{nA})$ NMDAR-mediated currents were detected as assessed by voltage-clamp recordings up to $4 \mathrm{~d}$ after injection (Fig. 1B). Similar results were obtained by replacing GluN2B CTD by the $\mathrm{r} 2$ motif at position GluN2B-S863 (GluN2Br2 subunit; Fig. $1 A, B$ ). In contrast, large currents (up to several $\mu \mathrm{A}$ ) were observed in control experiments with wt GluN2A or GluN2B subunits (Fig. 1B). The lack of current with retention tagged constructs was not due to a deficiency in subunit expression as revealed by Western-blot experiments (Fig. 1C). It is also unlikely to stem from truncation of the GluN2 CTD since complete deletion of the GluN1 and/or GluN2 CTD does not prevent functional expression (Vissel et al., 2002; Puddifoot et al., 2009; Maki et al., 2012; Punnakkal et al., 2012). Therefore, GluN1/ GluN2Ar1 and GluN1/GluN2Br2 diheteromers do not express at the plasma membrane, likely because they are retained in the ER. 
Table 1. Gating and pharmacological properties of diheteromeric and triheteromeric receptors

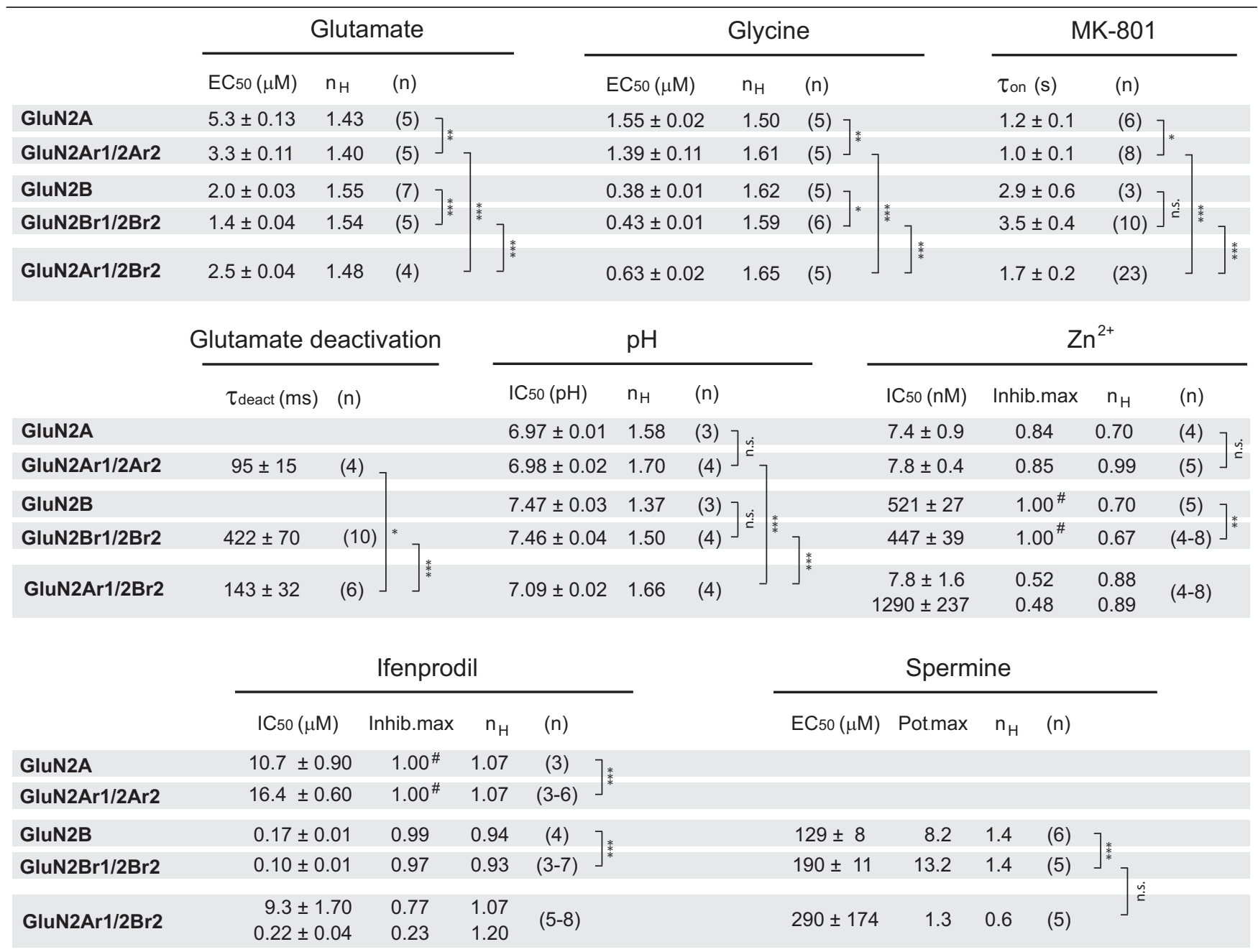

Values are related to the data shown in Figure 2. All receptors are composed of the GluN1-6A subunit. MK-801 $\tau$ on values are normalized relative to the mean value obtained for GluN1-6A/GluN2Ar1/GluN2Ar2 receptors. Note that while differences between GluN2Ar1/2Ar2 and GluN2A or GluN2Br1/2Br2 and GluN2B can be statistically significant, they are of small amplitude $\left(<1.8\right.$-fold change in EC $50, \mathrm{IC}_{50}, \tau_{\text {on }} \mathrm{MK}-801$ or $\tau_{\text {deact }}$ glutamate). \# fixed to 1 . ${ }^{* * *} p<0.001 ;{ }^{* *} p<$ $0.01 ;{ }^{*} p<0.05$, Bonferroni-corrected two-tailed post hoc $t$ test . ns, Not significant.

Interestingly, large currents indicating lack of proper ER retention were measured when the $\mathrm{r} 1$ or $\mathrm{r} 2$ motifs were introduced at other locations of the GluN2A or GluN2B CTD (see Materials and Methods). Together, these results indicate that the retention system derived from the GABA-B receptor is efficient in preventing NMDAR subunits from reaching the cell surface when incorporated at specific positions in the GluN2 CTD.

We next tested for expression of functional GluN1/GluN2A/ GluN2B triheteromers by coexpressing the three subunits GluN1, GluN2Ar1, and GluN2Br2. However, no NMDAR current could be measured in such conditions, suggesting that the expected release of ER retention by $\mathrm{r} 1 / \mathrm{r} 2$ recognition did not occur. Because the GluN1-1a subunit contains two strong ER retention motifs in the $\mathrm{C} 1$ cassette of its CTD (KKK and RRR; Horak and Wenthold, 2009), we reasoned that the close association of $\mathrm{r} 1$ and $\mathrm{r} 2$ may hinder proper GluN2-mediated ER release of the GluN1 subunit. We thus substituted both KKK and RRR motifs with alanines to escape GluN1 ER retention (Horak and Wenthold, 2009) and coexpressed GluN1-6A mutant subunit together with GluN2Ar1 and GluN2Br2. In such conditions, large NMDAR-mediated currents were measured, suggesting robust expression of functional GluN1/GluN2A/GluN2B triheteromers
(Fig. $1 B, C$ ). We confirmed that the measured currents were originating exclusively from triheteromers and not diheteromers by verifying that coinjection of GluN1-6A with GluN2Ar1 or GluN2Br2 resulted in lack of measurable NMDAR currents (despite subunit expression; Fig. $1 B, C$ ), as expected from the ER retention of the GluN2 subunit. In conclusion, the engineered $\mathrm{r} 1$ and $\mathrm{r} 2$ tags at the $\mathrm{C}$ terminus of GluN2A and GluN2B subunits allow expression of "pure" triheteromeric GluN1/GluN2A/ GluN2B receptors at the cell surface.

\section{Functional properties of triheteromeric \\ GluN1/GluN2A/GluN2B receptors}

We then characterized the gating and pharmacological properties of GluN1/GluN2A/GluN2B triheteromers. Because the GluN2 subunits are the major determinants of NMDAR functional diversity (Paoletti et al., 2013), triheteromers and diheteromers are expected to behave differently. We also checked that the introduced retention tags had minimal effect on receptor function by comparing the properties of "classical" diheteromers (containing two identical copies of a wt GluN2 subunit) with receptors composed of one r1-tagged GluN2A (or GluN2B) subunit and one r2-tagged GluN2A (or GluN2B) subunit (GluN1/GluN2Ar1/ 


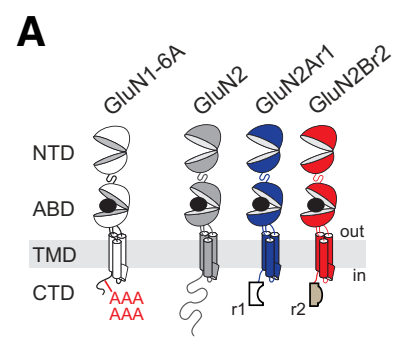

B

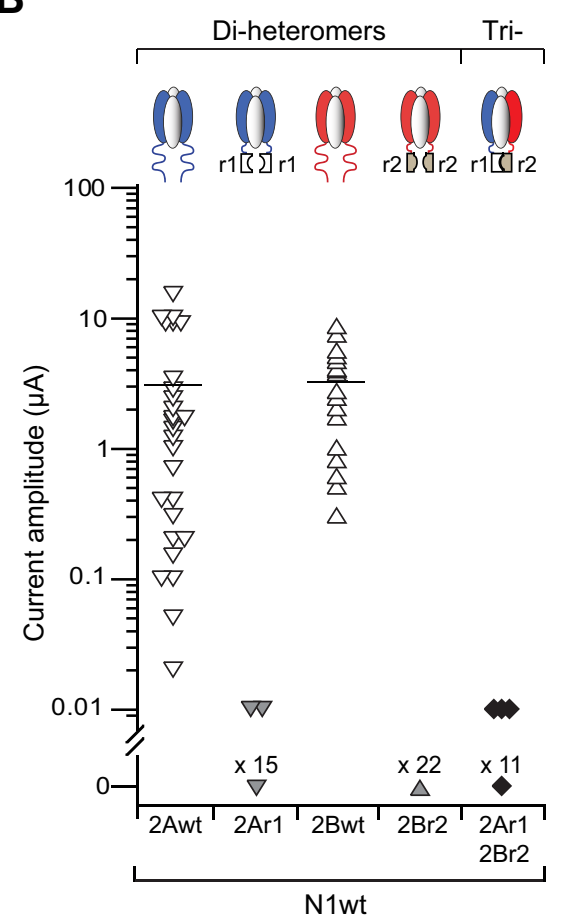

C

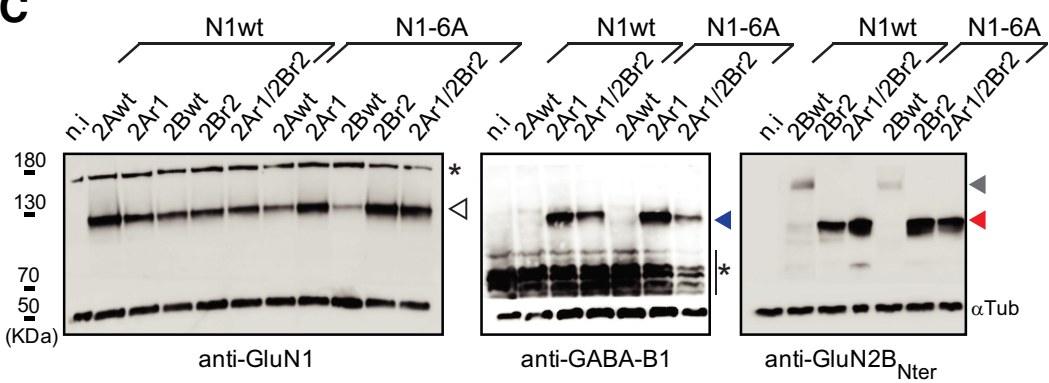

Di-

Tri-

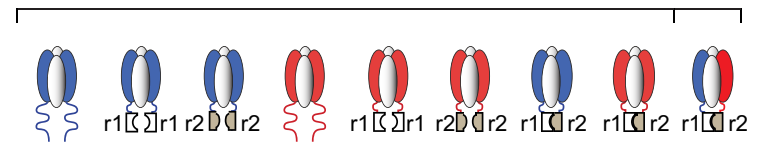

Figure 1. Selective expression of GluN1/GluN2A/GluN2B triheteromers at the surface of Xenopus oocytes. $A$, Schematic representation of the engineered NMDAR subunits. NTD, N-terminal domain; ABD, agonist-binding domain; TMD, transmembrane domain; CTD, C-terminal domain. The $r 1$ and $r 2$ tags contain the ER retention/retrieval signals derived from the GABA-B1 and GABA-B2 subunit, respectively. The GluN1-6A subunit has two endogenous ER retention signals $\left(\mathrm{K}_{875} \mathrm{KK}\right.$ and $\left.\mathrm{R}_{893} \mathrm{RR}\right)$ mutated into alanines. $\boldsymbol{B}$, Current amplitudes of various combinations of diheteromers and triheteromers. Currents were measured 3- $4 \mathrm{~d}$ after injection. Bars represent the average value for each condition. C, Immunoblots from oocytes expressing various combinations of $G$ luN1, GluN2A, and/or GluN2B subunits. The anti-GABA-B1 antibody is used to detect GluN2A subunits containing the r1 retention tag. The arrowheads indicate bands corresponding to GluN1wt of GluN1-6A (white, $\sim 115 \mathrm{kDa}$ ), GluN2Ar1 (blue, $\sim 110 \mathrm{kDa}$ ), GluN2B wt (gray, $\sim 180 \mathrm{kDa}$ ), and GluN2Br2 (red, $\sim 110 \mathrm{kDa}$ ). The stars indicate nonspecific bands. $\alpha$-tub, $\alpha$-Tubulin; and n.i., noninjected oocytes.

GluN2Ar2 and GluN1/GluN2Br1/GluN2Br2 receptors, respectively) (Table 1). We first measured the sensitivity to the agonists glutamate and glycine. We also estimated the channel maximal open probability $\left(P_{\mathrm{o}}\right)$ by measuring the kinetics of current inhibition by MK-801, a method classically used to index receptor channel $P_{\mathrm{o}}$ (Mony et al., 2011). GluN1/GluN2A/GluN2B triheteromers displayed sensitivity $\left(\mathrm{EC}_{50}\right)$ to glutamate and glycine intermediate to that of diheteromers (Fig. 2A, B, Table 1). Noticeably, whereas the sensitivity to glycine was close to that of GluN1/GluN2B receptors, sensitivity to glutamate was closer to that of GluN1/GluN2A receptors. Channel maximal $P_{\mathrm{o}}$ of triheteromers was significantly higher than that of GluN1/GluN2B diheteromers, reaching values close to the "high- $P_{\mathrm{o}}$ " GluN1/ GluN2A diheteromers (Fig. 2C). We also assessed glutamate deactivation kinetics, which dictates the time course of the EPSC, and found that GluN1/GluN2A/GluN2B triheteromers deactivate much faster than GluN1/GluN2B diheteromers and nearly as fast as GluN1/GluN2A diheteromers (Fig. 2D, Table 1).
A hallmark of NMDARs is their ability to be modulated by an array of small extracellular ligands, either synthetic or endogenous. Defining the sensitivity of triheteromers to these allosteric modulators is thus of major interest to understand receptor regulation and drug action. We first evaluated the sensitivity to extracellular protons, which exert potent tonic inhibition of NMDARs (Traynelis et al., 2010). Consistent with the strong influence of the GluN2A subunit on channel $P_{\mathrm{o}}$, the proton sensitivity of triheteromers was intermediate between that of diheteromers, but shifted toward that of GluN1/GluN2A receptors (Fig. 2E). We then investigated the sensitivity to zinc and ifenprodil, two subunit-specific allosteric inhibitors that are widely used to infer NMDAR subunit composition in native preparations (Paoletti et al., 2013). Zinc, at nanomolar concentrations, inhibits NMDARs endogenously by selectively binding to a site in the GluN2A N-terminal domain (NTD) (Vergnano et al., 2014). At higher concentrations, zinc inhibition of NMDARs also occurs through the GluN2B NTD (Rachline et al., 2005). Zinc sen- 
A

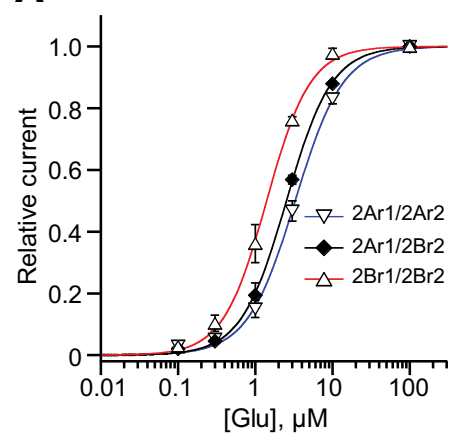

D

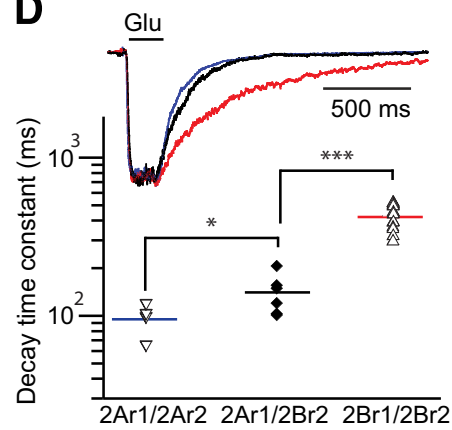

G

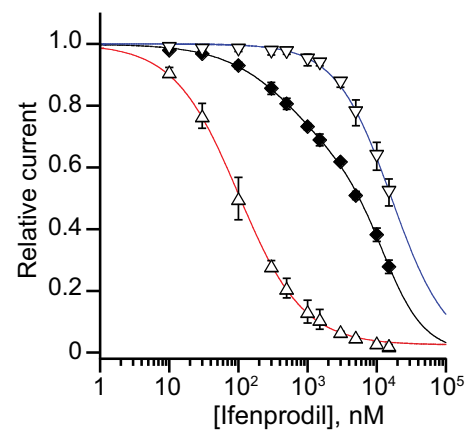

B

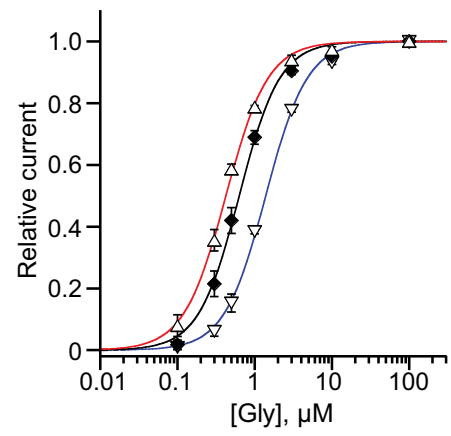

E

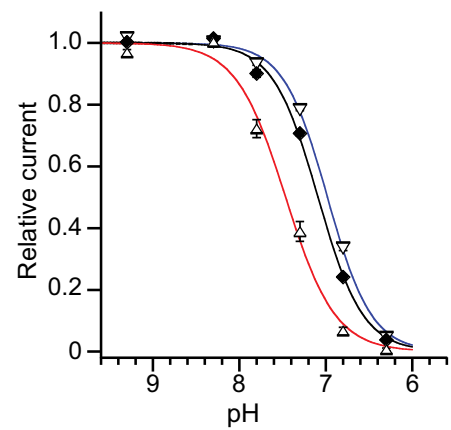

H

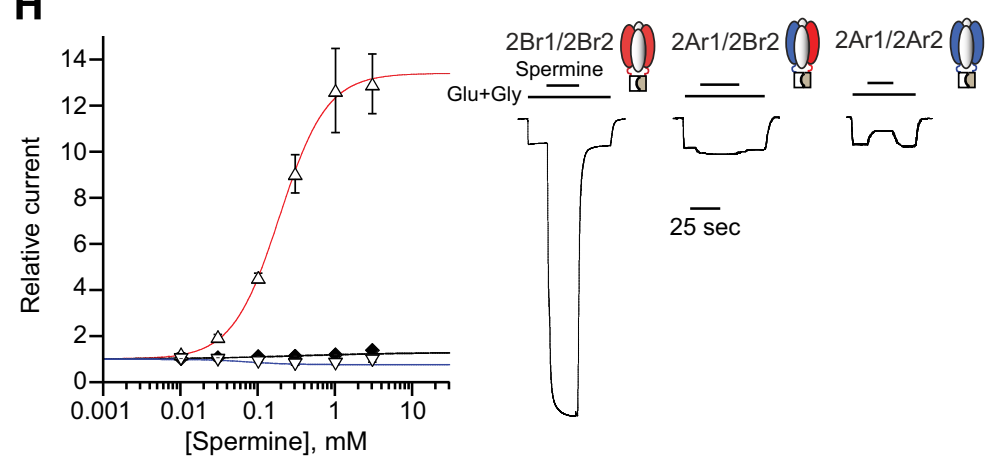

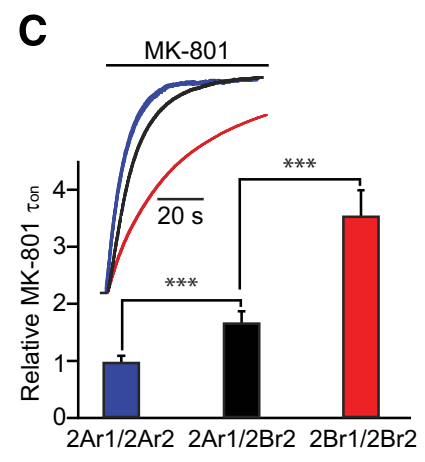

F

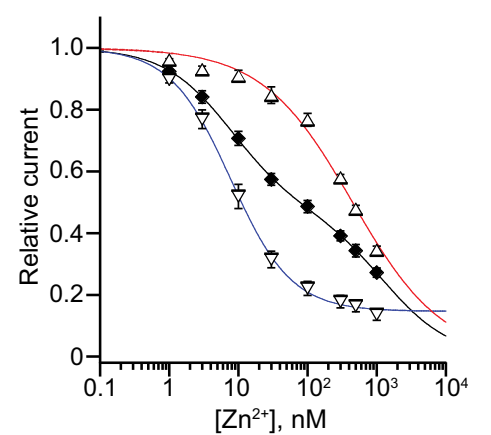

Figure 2. Functional properties of GluN1/GluN2A/GluN2B triheteromers. Recordings of GluN1-6A/GluN2Ar1/GluN2Ar2 (blue), GluN1-6A/GluN2Ar1/GluN2Br2 (black), and GluN1-6A/GluN2Br1/ GluN2Br2 (red) receptors. $\boldsymbol{A}$, Sensitivity to glutamate. $\boldsymbol{B}$, Sensitivity to glycine. $\boldsymbol{C}$, Inhibition kinetics by $10 \mathrm{~nm}$ MK-801. Inset, Normalized current traces. $\boldsymbol{D}$, Glutamate deactivation kinetics. Inset, Normalized current traces. $\boldsymbol{E}$, Sensitivity to protons. $\boldsymbol{F}$, Sensitivity to zinc. $\boldsymbol{G}$, Sensitivity to ifenprodil. $\boldsymbol{H}$, Sensitivity to spermine. Right, Current traces showing the effect of $100 \mu \boldsymbol{M}$ spermine application. Values of kinetics and dose-response curve fits are listed in Table 1. Error bars represent SD. ${ }^{*} p<0.05$; ${ }^{* * *} p<0.001$, Bonferroni-corrected two-tailed post hoc $t$ test.

sitivity of GluN1/GluN2A/GluN2B triheheteromers, which contain a single GluN2A NTD and a single GluN2B NTD, was intermediate between that of the two corresponding diheteromers (Fig. $2 F$ ). Interestingly, whereas the inhibition curves for diheteromers were satisfactorily fitted with a single component, the inhibition curve for triheteromers was clearly biphasic, with the component of highest affinity close to that of GluN2A diheteromers (Table 1). A biphasic curve was also observed when studying inhibition of triheteromers by ifenprodil, the prototypical GluN2B-selective antagonist acting at a GluN1-GluN2B NTD interface (Karakas et al., 2011). However, in contrast to zinc inhibition, the majority of the ifenprodil inhibition was accounted for by the low-affinity component (Fig. 2G, Table 1).

Finally, we evaluated the sensitivity of GluN1/GluN2A/ GluN2B triheteromers to spermine, an endogenous polyamine that selectively enhances GluN1/GluN2B diheteromers, presumably by binding an allosteric site located at a GluN1-GluN2B NTD dimer interface (Mony et al., 2011). Full spermine doseresponse curves revealed a striking loss-of-function phenotype for triheteromers. Indeed, whereas the spermine potentiation was massive at GluN1/GluN2B diheteromers ( $>12$-fold maximal current increase), it was greatly attenuated at triheteromers $(\sim 1.2$; Fig. $2 H$, Table 1$)$. Therefore, two copies of the GluN2B subunits are required for polyamines to exert their full potentiation at NMDARs.

\section{Influence of the GluN1 CTD on triheteromeric receptor expression}

The GluN1-1a subunit traffics poorly to the plasma membrane, whereas other GluN1 CTD splice variants go readily to the cell surface (Horak and Wenthold, 2009). Because cell surface expression of triheteromers required suppression of two ER retention motifs in the GluN1-1a CTD (see Selective expression of triheteromeric NMDARs), we reasoned that the GluN1-2a, GluN1-3a, or GluN1-4a subunits in their wt versions may permit cell surface expression of triheteromers. Large currents were indeed recorded when oocytes were coinjected with GluN2Ar1, GluN2Br2, and either one of the three GluN1 splice variants. 

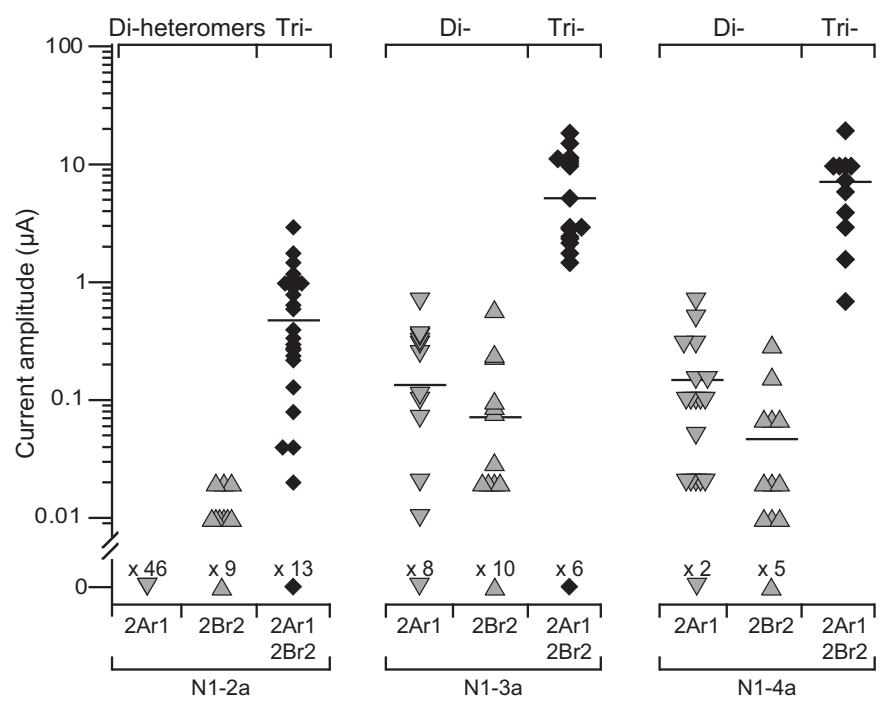

Figure 3. Influence of GluN1 subunit isoform on triheteromer expression. Current amplitudes of various diheteromers and triheteromers composed of the indicated GluN1 and GluN2 subunits. The bars represent the average current value for each condition.

However, control experiments revealed that, in the case of GluN1-3a and GluN1-4a, proper intracellular retention of diheteromers (GluN1/GluN2Ar1 and GluN1/GluN2Br2) was not achieved (Fig. 3). In contrast, in the case of GluN1-2a, diheteromer "leakage" was negligible (currents $\leq 20 \mathrm{nA}$ ), indicating that currents measured after coexpression of GluN1-2a, GluN2Ar1, and GluN2Br2 were almost entirely carried by triheteromers with minimal contribution from diheteromers (Fig. 3). A similar pattern was observed with a CTD-truncated GluN1 subunit (GluN1 $\Delta$ C), highlighting the critical importance of GluN1 CTD in the intracellular trafficking of the triheteromers.

\section{Discussion}

By engineering GluN2 subunits with imported retention signals from GABA-B receptors, we have developed a strategy allowing selective expression of triheteromeric NMDARs in Xenopus oocytes. Our work follows a study that used a similar approach to isolate triheteromeric NMDARs (Hansen et al., 2014), although the two studies differ in the exact GluN2 constructs and functional description of GluN1/GluN2A/GluN2B triheteromers.

In agreement with Hansen et al. (2014), our data show that the GABA-B retention system provides a powerful means with which to constrain subunit composition of heterologously expressed NMDARs. This system enables exclusive cell surface expression of triheteromeric NMDARs, the coexpressed diheteromers being retained in the ER. Importantly, the presence of modified GluN2 CTDs with engineered peptide tags has no (or minimal) effect on the functional properties of NMDARs, in agreement with minimal perturbation of the receptor gating core (see also Hansen et al., 2014). We also show that the position and amino acid environment of the retention tags in the GluN2 CTDs is critical for the system to work. Our retention tags were placed close to the transmembrane pore to avoid possible cross talk between receptors that would allow diheteromers to escape the ER. In contrast, Hansen et al. (2014) inserted the retention tags at the very C terminus and used a chimeric GluN2B subunit with its CTD swapped for that of GluN2A. Differences between the two studies also extend to the type of GluN1 subunit. Although Hansen et al. (2014) used the classic wt GluN1-1a isoform, our approach relies

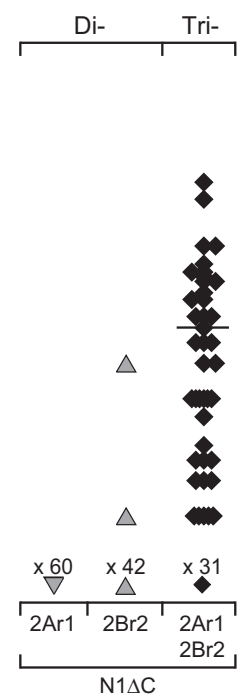

on GluN1 variants that are not ER retained, such as the wt GluN1-2a subunit, a GluN1 isoform abundantly expressed in the CNS (Laurie and Seeburg, 1994).

By recording from Xenopus oocytes selectively expressing triheteromers at their surface, we establish that GluN1/GluN2A/ GluN2B triheteromers have unique gating and pharmacological properties. Our data reveal that the GluN2A subunit has a dominant effect on properties as diverse as channel $P_{\mathrm{o}}$, glutamate sensitivity, and deactivation kinetics, as well as allosteric modulation by $\mathrm{H}^{+}$, ifenprodil, and polyamines, modulators that bind various sites on the receptor. In contrast, the GluN2B phenotype dominates for glycine sensitivity, whereas for zinc sensitivity, the phenotype is intermediate between GluN2A and GluN2B, as revealed by the comparable contribution of the high- and low-affinity components to triheteromer inhibition. The asymmetric contribution of each GluN2 subunit to specific functions of the triheteromers raises fundamental questions that remain to be addressed about subunit coupling and domain interactions in the tetrameric receptor complex. We note that our present results on GluN1/GluN2A/GluN2B triheteromers are consistent with our previous findings on triheteromer pharmacology obtained using a completely different approach (Hatton and Paoletti, 2005), thus lending support to the validity of our retention signal approach. In particular, we confirm that diheteromers and triheteromers differ in their sensitivity to zinc and ifenprodil, with triheteromers retaining high potency but reduced efficacy. In contrast, Hansen et al. (2014) conclude that zinc (but not ifenprodil) inhibits diheteromers and triheteromers similarly. Because triheteromers contain a single high-affinity binding site for zinc or ifenprodil whereas diheteromers have two (one per GluN1/GluN2 NTD heterodimer; Karakas et al., 2011), stronger inhibition at diheteromers might be expected and likely reflects concerted action of the two "active" (i.e., ligand-bound) NTD heterodimers on the downstream gating machinery. Consistent with this, we find that two "active" NTD polyaminebinding sites are also required for polyamines to exert full potentiation of GluN2B-containing receptors.

By unveiling novel aspects about the properties of one of the most abundant NMDAR subtypes in the adult brain, our work adds significantly to our understanding of NMDAR functional and pharmacological diversity. It also provides an easy-to-use and efficient strategy to express a homogeneous population of triheteromeric NMDARs at the cell surface of Xenopus oocytes. This strategy should permit characterizing other triheteromeric combinations, such as GluN1/GluN2A/GluN2C, GluN1/GluN2B/ GluN2D, or GluN3-containing receptors, which populate various brain regions but remain ill-defined functionally (SanzClemente et al., 2013; Paoletti et al., 2013). It should also help to define the properties of mutant NMDARs composed of two GluN2 subunits differing by just one (or a few) mutations, as occur in patients carrying heterozygous disease-causing mutations in NMDAR genes (Soto et al., 2014). More generally, by constraining subunit stoichiometry, the retention signal approach has the potential to allow specific expression of a variety of other iGluRs sharing the dimer-of-dimer structure of NMDARs, 
such as heteromeric AMPA GluA1/GluA2 receptors or kainate GluK2/GluK5 receptors. Because these receptors are preferentially expressed in vivo (Herguedas et al., 2013; Kumar and Mayer, 2013), important implications for our understanding of neurotransmission and for drug discovery are to be expected.

\section{References}

Al-Hallaq RA, Conrads TP, Veenstra TD, Wenthold RJ (2007) NMDA diheteromeric receptor populations and associated proteins in rat hippocampus. J Neurosci 27:8334-8343. CrossRef Medline

Brock C, Oueslati N, Soler S, Boudier L, Rondard P, Pin JP (2007) Activation of a dimeric metabotropic glutamate receptor by intersubunit rearrangement. J Biol Chem 282:33000-33008. CrossRef Medline

Calver AR, Robbins MJ, Cosio C, Rice SQ, Babbs AJ, Hirst WD, Boyfield I, Wood MD, Russell RB, Price GW, Couve A, Moss SJ, Pangalos MN (2001) The C-terminal domains of the GABA(b) receptor subunits mediate intracellular trafficking but are not required for receptor signaling. J Neurosci 21:1203-1210. Medline

Chazot PL, Stephenson FA (1997) Molecular dissection of native mammalian forebrain NMDA receptors containing the NR1 C2 exon: direct demonstration of NMDA receptors comprising NR1, NR2A, and NR2B subunits within the same complex. J Neurochem 69:2138-2144. Medline

Gielen M, Le Goff A, Stroebel D, Johnson JW, Neyton J, Paoletti P (2008) Structural rearrangements of NR1/NR2A NMDA receptors during allosteric inhibition. Neuron 57:80-93. CrossRef Medline

Gray JA, Shi Y, Usui H, During MJ, Sakimura K, Nicoll RA (2011) Distinct modes of AMPA receptor suppression at developing synapses by GluN2A and GluN2B: single-cell NMDA receptor subunit deletion in vivo. Neuron 71:1085-1101. CrossRef Medline

Hansen KB, Ogden KK, Yuan H, Traynelis SF (2014) Distinct functional and pharmacological properties of Triheteromeric GluN1/GluN2A/ GluN2B NMDA receptors. Neuron 81:1084-1096. CrossRef Medline

Hatton CJ, Paoletti P (2005) Modulation of triheteromeric NMDA receptors by N-terminal domain ligands. Neuron 46:261-274. CrossRef Medline

Herguedas B, Krieger J, Greger IH (2013) Receptor heteromeric assemblyhow it works and why it matters: the case of ionotropic glutamate receptors. Prog Mol Biol Transl Sci 117:361-386. CrossRef Medline

Horak M, Wenthold RJ (2009) Different roles of C-terminal cassettes in the trafficking of full-length NR1 subunits to the cell surface. J Biol Chem 284:9683-9691. CrossRef Medline

Karakas E, Simorowski N, Furukawa H (2011) Subunit arrangement and phenylethanolamine binding in GluN1/GluN2B NMDA receptors. Nature 475:249-253. CrossRef Medline

Kumar J, Mayer ML (2013) Functional insights from glutamate receptor ion channel structures. Annu Rev Physiol 75:313-337. CrossRef Medline

Laurie DJ, Seeburg PH (1994) Regional and developmental heterogeneity in splicing of the rat brain NMDAR1 mRNA. J Neurosci 14:3180-3194. Medline

Luo J, Wang Y, Yasuda RP, Dunah AW, Wolfe BB (1997) The majority of $\mathrm{N}$-methyl-D-aspartate receptor complexes in adult rat cerebral cortex contain at least three different subunits (NR1/NR2A/NR2B). Mol Pharmacol 51:79-86. Medline

Maki BA, Aman TK, Amico-Ruvio SA, Kussius CL, Popescu GK (2012) $\mathrm{C}$-terminal domains of $\mathrm{N}$-methyl-D-aspartic acid receptor modulate unitary channel conductance and gating. J Biol Chem 287:36071-36080. CrossRef Medline
Margeta-Mitrovic M, Jan YN, Jan LY (2000) A trafficking checkpoint controls GABA(B) receptor heterodimerization. Neuron 27:97-106. CrossRef Medline

Mony L, Zhu S, Carvalho S, Paoletti P (2011) Molecular basis of positive allosteric modulation of GluN2B NMDA receptors by polyamines. EMBO J 30:3134-3146. CrossRef Medline

Pagano A, Rovelli G, Mosbacher J, Lohmann T, Duthey B, Stauffer D, Ristig D, Schuler V, Meigel I, Lampert C, Stein T, Prezeau L, Blahos J, Pin J, Froestl W, Kuhn R, Heid J, Kaupmann K, Bettler B (2001) C-terminal interaction is essential for surface trafficking but not for heteromeric assembly of GABA(b) receptors. J Neurosci 21:1189-1202. Medline

Paoletti P, Ascher P, Neyton J (1997) High-affinity zinc inhibition of NMDA NR1-NR2A receptors. J Neurosci 17:5711-5725. Medline

Paoletti P, Bellone C, Zhou Q (2013) NMDA receptor subunit diversity: impact on receptor properties, synaptic plasticity and disease. Nat Rev Neurosci 14:383-400. CrossRef Medline

Puddifoot CA, Chen PE, Schoepfer R, Wyllie DJ (2009) Pharmacological characterization of recombinant NR1/NR2A NMDA receptors with truncated and deleted carboxy termini expressed in Xenopus laevis oocytes. Br J Pharmacol 156:509-518. CrossRef Medline

Punnakkal P, Jendritza P, Köhr G (2012) Influence of the intracellular GluN2 C-terminal domain on NMDA receptor function. Neuropharmacology 62:1985-1992. CrossRef Medline

Rachline J, Perin-Dureau F, Le Goff A, Neyton J, Paoletti P (2005) The micromolar zinc-binding domain on the NMDA receptor subunit NR2B. J Neurosci 25:308-317. CrossRef Medline

Rauner C, Köhr G (2011) Triheteromeric NR1/NR2A/NR2B receptors constitute the major $\mathrm{N}$-methyl-D-aspartate receptor population in adult hippocampal synapses. J Biol Chem 286:7558-7566. CrossRef Medline

Ryan TJ, Emes RD, Grant SG, Komiyama NH (2008) Evolution of NMDA receptor cytoplasmic interaction domains: implications for organisation of synaptic signalling complexes. BMC Neurosci 9:6. CrossRef Medline

Sanz-Clemente A, Nicoll RA, Roche KW (2013) Diversity in NMDA receptor composition: many regulators, many consequences. Neuroscientist 19:62-75. CrossRef Medline

Sheng M, Cummings J, Roldan LA, Jan YN, Jan LY (1994) Changing subunit composition of heteromeric NMDA receptors during development of rat cortex. Nature 368:144-147. CrossRef Medline

Soto D, Altafaj X, Sindreu C, Bayés A (2014) Glutamate receptor mutations in psychiatric and neurodevelopmental disorders. Commun Integr Biol 7:e27887. CrossRef Medline

Tovar KR, McGinley MJ, Westbrook GL (2013) Triheteromeric NMDA receptors at hippocampal synapses. J Neurosci 33:9150-9160. CrossRef Medline

Traynelis SF, Wollmuth LP, McBain CJ, Menniti FS, Vance KM, Ogden KK, Hansen KB, Yuan H, Myers SJ, Dingledine R (2010) Glutamate receptor ion channels: structure, regulation, and function. Pharmacol Rev 62:405496. CrossRef Medline

Vergnano AM, Rebola N, Savtchenko LP, Pinheiro PS, Casado M, Kieffer BL, Rusakov DA, Mulle C, Paoletti P (2014) Zinc dynamics and action at excitatory synapses. Neuron 82:1101-1114. CrossRef Medline

Vissel B, Krupp JJ, Heinemann SF, Westbrook GL (2002) Intracellular domains of NR2 alter calcium-dependent inactivation of N-methyl-Daspartate receptors. Mol Pharmacol 61:595-605. CrossRef Medline

Zhu S, Stroebel D, Yao CA, Taly A, Paoletti P (2013) Allosteric signaling and dynamics of the clamshell-like NMDA receptor GluN1 N-terminal domain. Nat Struct Mol Biol 20:477-485. CrossRef Medline 